\title{
The association between cystatin $C$ and COPD: a meta-analysis and systematic review
}

Limin Chai, Wei Feng, Cui Zhai, Wenhua Shi, Jian Wang, Xin Yan, Qingting Wang, Qianqian Zhang and Manxiang $\mathrm{Li}^{*}$

\begin{abstract}
Background: In recent years, many studies have discovered that cystatin C (Cys C) may play an important role in respiratory diseases, especially in chronic obstructive pulmonary disease (COPD). However, the findings of these studies were inconsistent. This systematic review and meta-analysis aimed to assess the relationship between serum Cys $C$ and COPD.

Methods: We conducted a systematic literature search in PubMed, Embase, Web of Science, Wanfang databases, and the China National Knowledge Infrastructure. The standardized mean difference (SMD), Fisher's Z-value and 95\% confidence interval (Cl) were calculated to investigate the effect sizes. Subgroup analyses were performed on disease status, ethnicity, assay method, and study design. Sensitivity was performed, and publication bias was assessed.

Results: A total of 15 studies, including 4079 COPD patients and 5949 controls, were included in this meta-analysis. The results showed that serum Cys $C$ levels in patients with COPD were significantly higher than those in controls $(\mathrm{SMD}=0.99,95 \% \mathrm{Cl}=0.62-1.37, P<0.001)$, especially in AECOPD (SMD $=1.59,95 \% \mathrm{Cl}=1.05-2.13, P<0.001)$, and there were statistically different among AECOPD and SCOPD (SMD $=0.35,95 \% \mathrm{Cl}=0.10-0.59, P=0.005)$. The serum Cys $C$ levels were negatively correlated with FEV1\%pre $(Z=-0.45,95 \% \mathrm{Cl}=-0.58--0.32, P=0.011)$ and FEV1/FVC $(Z=-0.32,95 \% C l=-0.50--0.14, P=0.006)$. The serum Cys $C$ levels were independent of ethnicity, assay method, and study design.
\end{abstract}

Conclusion: Serum Cys C levels were associated with COPD and COPD exacerbation, and they were inversely correlated with FEV1\%pre and FEV1/FVC.

Keywords: Cys C, COPD, Exacerbation, Meta-analysis

\section{Background}

Chronic obstructive pulmonary disease (COPD) is a disease characterized by incomplete reversibility of airflow obstruction, chronic inflammation of the airways, and systemic effects or comorbidities [1-3]. Although COPD

\footnotetext{
* Correspondence: manxiangli@hotmail.com

Department of Respiratory and Critical Care Medicine, the First Affiliated Hospital of Xi'an Jiaotong University, No. 277, West Yanta Road, Xi'an 710061, Shaanxi, People's Republic of China
}

is a preventable and treatable disease, it still brings heavy economic burden to family and the society [4-6]. However, the pathogenesis of COPD is not completely elucidated, and it is the long-term interaction of many factors including environmental factors and genetic factors [7]. Nowadays, GOLD guidelines recommend using spirometry assessment as a diagnostic and prognostic indicator for COPD [8]. However, fewer than half of the patients have the data from spirometry lung function tests $[9$,

C C The Author(s). 2020 Open Access This article is licensed under a Creative Commons Attribution 4.0 International License, which permits use, sharing, adaptation, distribution and reproduction in any medium or format, as long as you give appropriate credit to the original author(s) and the source, provide a link to the Creative Commons licence, and indicate if changes were made. The images or other third party material in this article are included in the article's Creative Commons licence, unless indicated otherwise in a credit line to the material. If material is not included in the article's Creative Commons licence and your intended use is not permitted by statutory regulation or exceeds the permitted use, you will need to obtain permission directly from the copyright holder. To view a copy of this licence, visit http://creativecommons.org/licenses/by/4.0/ The Creative Commons Public Domain Dedication waiver (http://creativecommons.org/publicdomain/zero/1.0/) applies to the data made available in this article, unless otherwise stated in a credit line to the data. 
10]. Therefore, it is of great significance to explore simple and novel biomarkers of COPD for the early diagnosis and monitoring of prognosis.

Cystatin C (Cys C), an inhibitor of cysteine proteinase, belongs to the member of family II of the cystatin superfamily [11]. Cys $C$ is an alkaline secreted protein, with a molecular mass of $13.3 \mathrm{kDa}$, which widely exists in various tissues of nucleated cells and body fluids, and produced by the body's all nucleated cells [12, 13]. Cys C can protect human cells from improper hydrolysis of proteases inside and outside the body. Previous researches suggest that serum Cys $C$ is entirely determined by the glomerular filtration rate (GFR), and may more accurately estimate GFR than creatinine [14-16]. It has been suggested that Cys $C$ is valuable in predicting cardiovascular mortality in cardiovascular disease [17, 18]. In recent years, there are many studies on the clinical significance of serum Cys $C$ levels changes in respiratory diseases. It has been studied that Cys $\mathrm{C}$ plays a role in pathogenesis of obstructive sleep apnea syndrome [1020], lung malignancy [21], pleural effusions [22], and emphysema [23].

Recent studies indicate a possible link between Cys C and COPD [24-28]; however, the conclusion is not clear. Most studies have shown that serum Cys $\mathrm{C}$ levels are higher in the exacerbation group than stable COPD or healthy control $[29,30]$, but Selder et al. [31] find that serum Cys $C$ levels are lower in exacerbation group than stable COPD. We performed this meta-analysis to clarify the associations between serum Cys $C$ levels and COPD as well as COPD exacerbation. This study might provide new perspectives in explaining the relationship between Cys $\mathrm{C}$ and COPD, COPD exacerbation.

\section{Methods}

\section{Literature search strategy}

We had searched PubMed, Embase, Web of Science, Wanfang databases (www.wanfangdata.com.cn), and the China National Knowledge Infrastructure (CNKI, www. cnki.net) to collect articles involving the role of Cys $\mathrm{C}$ in COPD, and the retrieval time was before Jul 312,019. The keywords used for searching were: ("Cystatin C" or "Cys C") in combination with ("chronic obstructive pulmonary disease" or "COPD"). Besides, the reference lists of all selected articles were manually searched for further potentially eligible articles. There were no restrictions on language, population, publication date, or type of report, and unpublished data were excluded.

\section{Inclusion and exclusion criteria}

All the potential studies were independently selected by two reviewers (Limin Chai and Wei Feng) based on the following inclusion and exclusion criteria. The researches in the meta-analysis accorded with the following inclusion criteria: 1) a study involving the role of Cys C in COPD; 2) cohort, case-control, or cross-section design; 3) if there was duplication of data, only the most complete and recent studies were included; and 4) the effect size and its 95\% confidence interval $(\mathrm{CI})$ were provided or could be estimated. Furthermore, the following exclusion criteria were used: 1) not related to Cys C or COPD; 2) Based on family or sibling pairs studies; 3 ) measured Cys $\mathrm{C}$ concentrations in biological samples other than blood, including urine, sputum, or BALF; 4) reviews, reports, comments, letters, meta-analysis, abstracts etc. were also excluded.

\section{Quality of the literature}

The Newcastle-Ottawa scale (NOS) was used to assess the quality of eligible studies from three aspects: (1) selection of cases and controls; (2) comparability between cases and controls; (3) exposure in cases and controls. The NOS has a score range of zero to nine, and studies with a score of more than seven were thought to be of high quality [32].

\section{Data extraction}

Two evaluators extracted the data independently and used a standardized form. Collected the following items: the name of the first author, year of publication, study design, country and ethnicity of participants, sample, method of Cys $\mathrm{C}$ measurement, sample size, serum Cys $\mathrm{C}$ levels, age of participant. The quality of each selected study was also independently assessed by two reviewers who used the Newcastle-Ottawa Scale. Discussion with another investigator resolved it when they had divergences.

\section{Statistical analysis}

In this study, all the statistical analyses were carried out using STATA 12.0 software (Stata Corp LP, College Station, TX, the United States). For the continuous data, the standardized mean difference (SMD) and 95\% CI were calculated. In the case of only reporting SEM, SD was estimated by the formula: $\mathrm{SD}=\mathrm{SEM} \times$ Sqrt (sample size) www.cochranehandbook.org.). Estimating the sample mean and standard deviation from the sample size, median, range and/or interquartile range [33]. $P<0.05$ indicated the statistically significant. Heterogeneity among studies was assessed based on the chi-square Q test and $\mathrm{I} 2$ test. Heterogeneity was significant when the $P<0.1$ or $\mathrm{I}^{2}>50 \%$. When there was no heterogeneity, the fixed effect model was used for analysis. Otherwise, the random effect model was used. In addition, Fisher's r-to-Z transformation was used to convert each correlation coefficient into their approximately associated $Z$ statistics. If the Fisher's Z-value could not be directly obtained, which is considered the normal distribution [34]. Moreover, Begg's tests and Egger's tests were used to evaluating publication bias $[35,36]$. Meta-regression 
analysis and subgroup analysis based on ethnicity, assay method, and study design were used to explore the potential sources of heterogeneity. Sensitivity analysis was applied by performing leave-one-out function to test the robustness of the pooled estimate.

\section{Results}

\section{Characteristics of included studies}

The literature screening process and results were shown in Fig. 1. Briefly, a total of 186 articles were preliminarily distinguished from PubMed $(n=22)$, Embase $(n=55)$, web of science $(n=27)$, Wanfang $(n=32)$, and China National Knowledge Infrastructure (CNKI) $(n=50)$, among which 53 were duplicates. After screening on title and abstract, 25 articles remained for full text review. In consequence, 15 studies fulfilled the inclusion criteria, involving 4079 cases and 5949 controls. The studies, which were included in this meta-analysis, were published between 2012 and 2019 and were from the USA, Japan, Turkey, China, and Spain. The information of these studies was extracted and listed separately in the Table 1. Three articles (20\%) were of moderate quality, and the other included studies $(80 \%)$ were of high quality according to the NOS quality score evaluation.

\section{Serum Cys $\mathrm{C}$ levels in patients with COPD}

To investigate potential correlations of serum Cys C levels with COPD, fifteen studies were enrolled for meta-analyses. We analyzed the heterogeneity of COPD vs control for the 12 studies, and the value of $\mathrm{I}^{2}$ value was $95.2 \%$ and $P<0.001$. A heterogeneity was observed between these studies; therefore, the random-effects model was used for synthesis of the data. The pooled effect sizes indicated that the serum Cys $C$ levels in patients with COPD were significantly higher than that in control $(\mathrm{SMD}=0.99,95 \% \mathrm{CI}=0.62-1.37, P<0.001$; Fig. 2). Moreover, we conducted a multivariate metaregression analysis to explore the possible confounding factors leading to heterogeneity. The results showed that the year of publication, the case group, the proportion of cases and control, the ratio of men in cases and control, and the study quality as confounding factors had no significant effect on heterogeneity ( $P$ values were 0.589 , $0.229,0.480,0.895$ and 0.066 ). We further conducted a sensitivity analysis by successively extracting each study from this meta-analysis, which did not change the direction or statistical significance of SMD, indicating that this meta-analysis was stable (Fig. 3). In addition, Begg's and Egger's tests were used to evaluate publication bias, Begg's test found no publication bias $(P=0.373)$, and publication bias was found by Egger's tests $(P=0.007)$, therefore the trim and fill analysis was further performed and showed no further studies required [47].

\section{Subgroup meta-analysis}

Due to the significant heterogeneity, we further performed subgroup analyses base on disease status, ethnicity, assay method, and study design. In the subgroup analysis of disease status (acute exacerbation COPD (AECOPD) vs control, stable COPD (SCOPD) vs control, AECOPD vs SCOPD) (Table 2), the meta-analysis results indicated that they were statistically different between AECOPD and

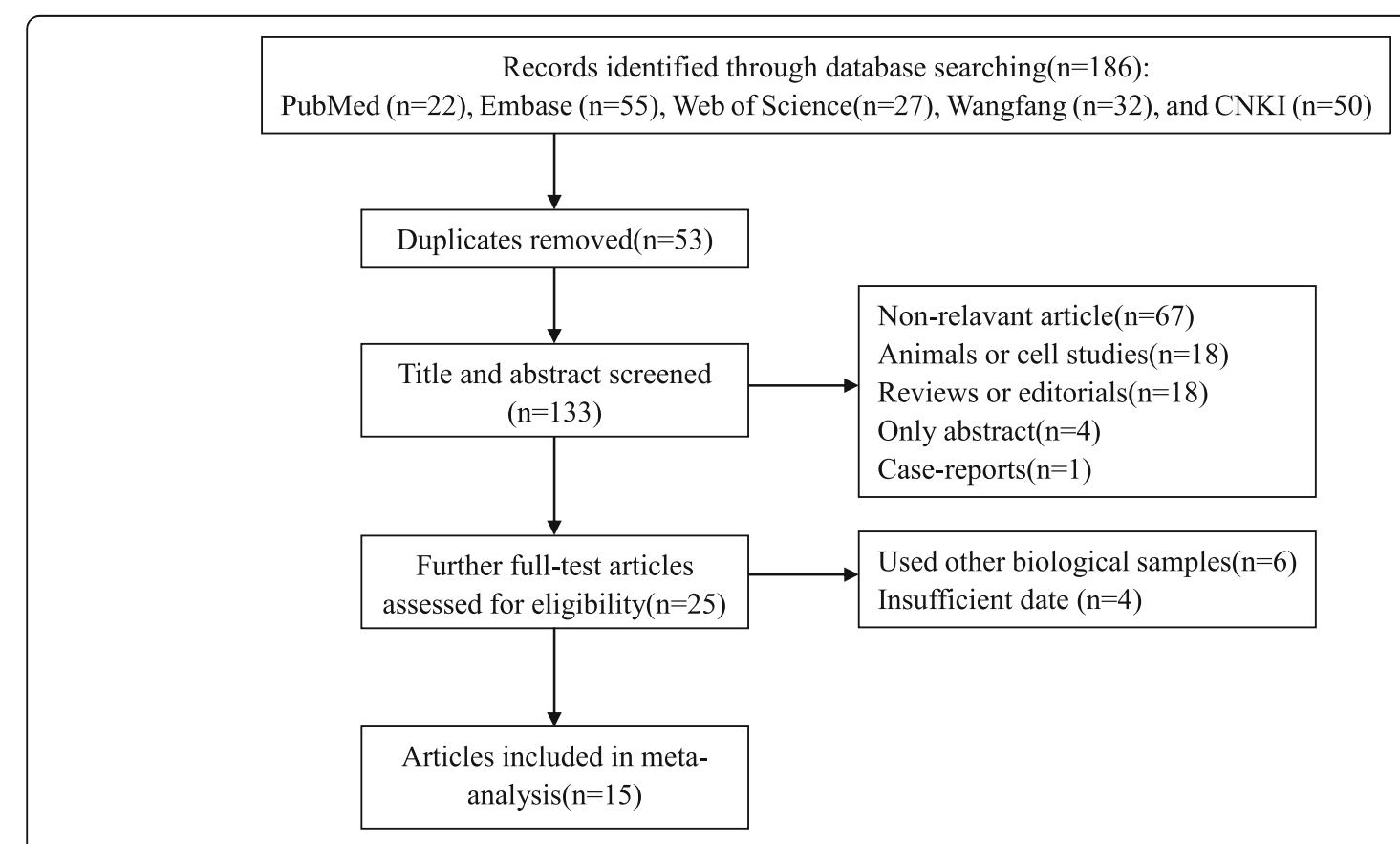

Fig. 1 Flow chart of included/excluded studies using the criteria before the meta-analysis. Abbreviations: CNKI, China National Knowledge Infrastructure 


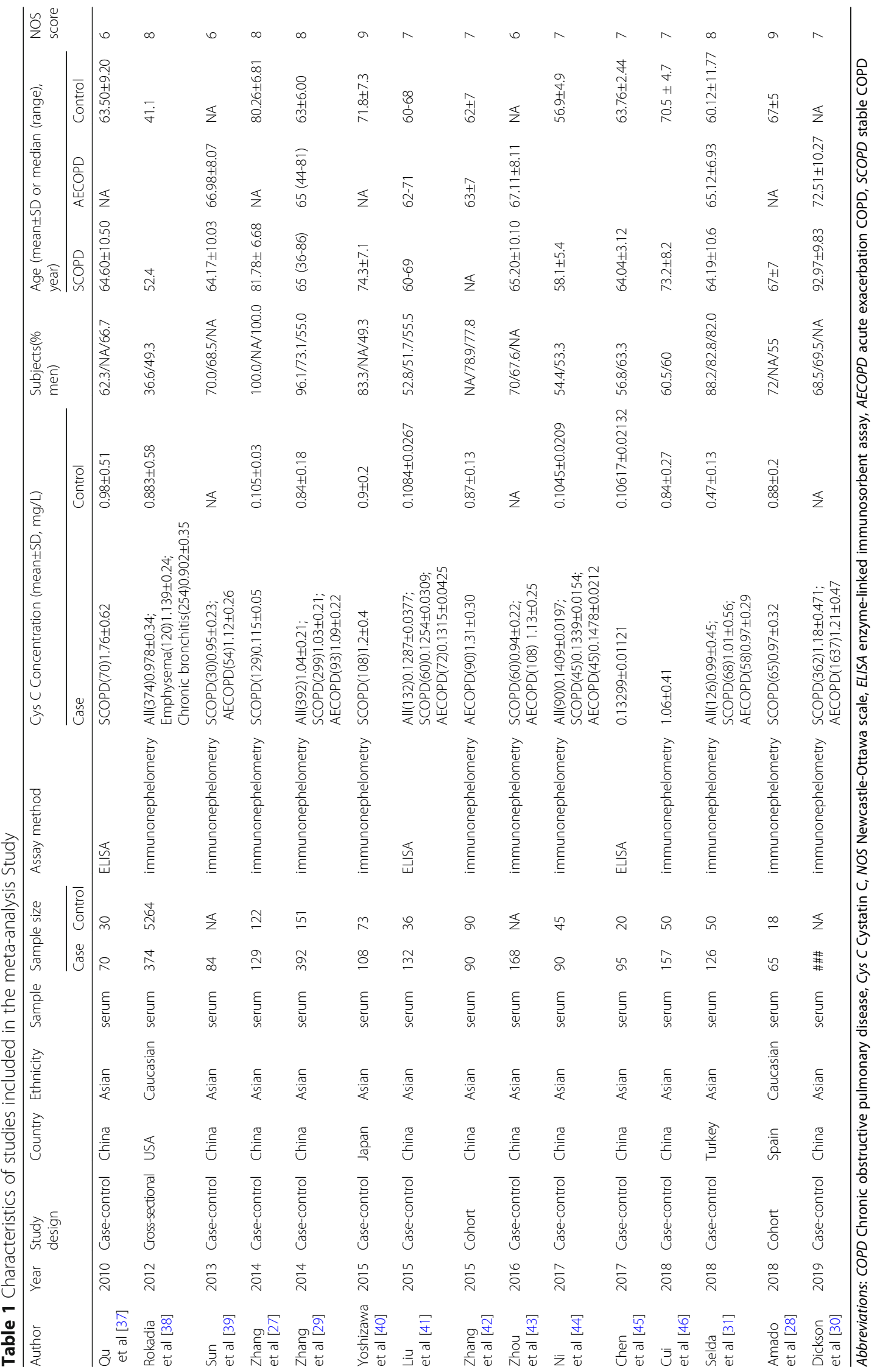




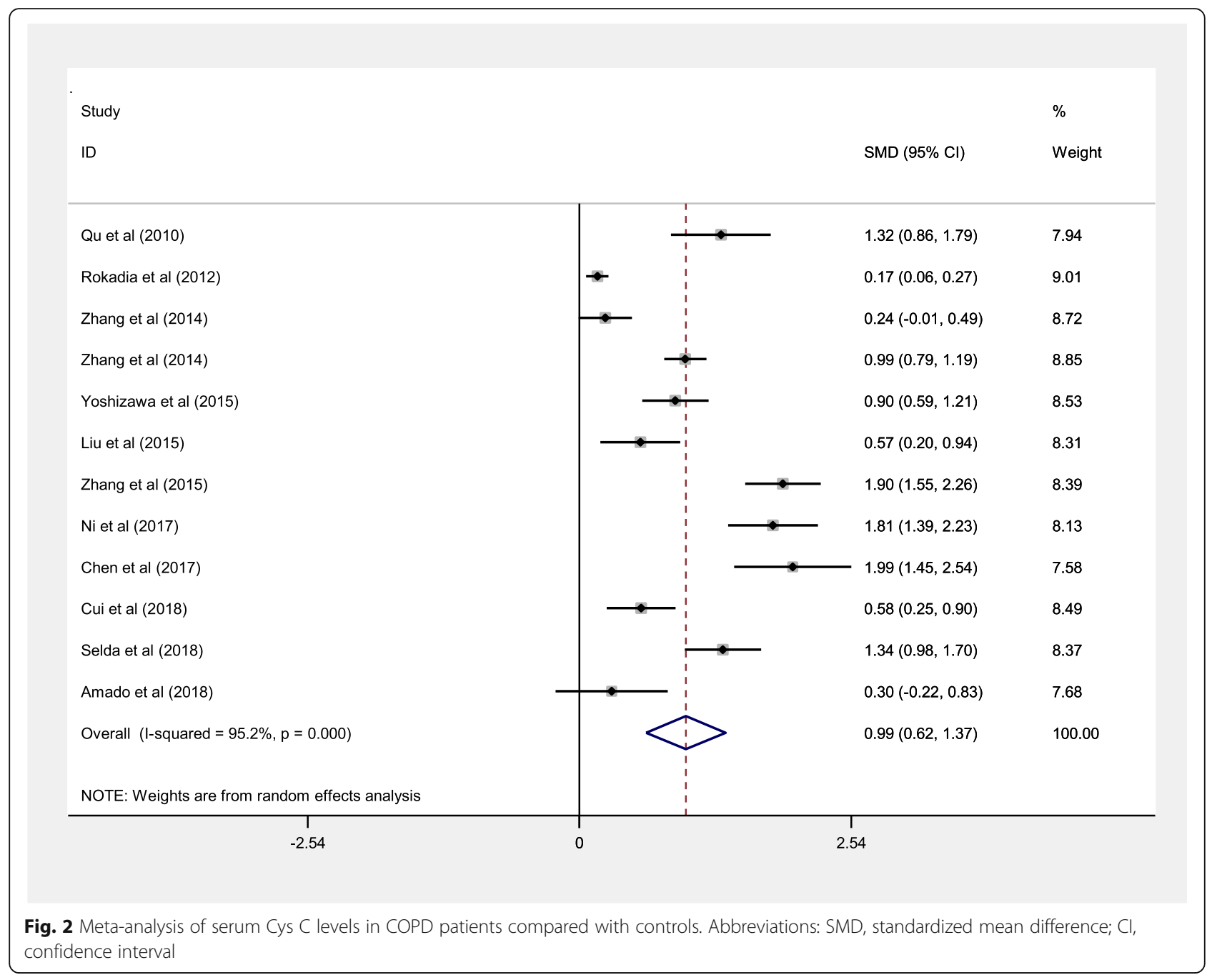

controls $\quad(\mathrm{SMD}=1.59, \quad 95 \% \mathrm{CI}=1.05-2.13, \quad P<0.001$, Fig. 4), SCOPD and controls $(\mathrm{SMD}=0.88,95 \% \mathrm{CI}=$ $0.56-1.20, P<0.001), \mathrm{AECOPD}$ and SCOPD $(\mathrm{SMD}=$ $0.35,95 \% \mathrm{CI}=0.10-0.59, \quad P=0.005)$. The ethnicityspecial subgroup analyses showed that COPD among the Caucasians had lower serum Cys C levels $(\mathrm{SMD}=$ $0.17,95 \% \mathrm{CI}=0.07-0.28, P=0.001$, Fig. $5 \mathrm{a})$ than subjects in COPD among the Asians $(\mathrm{SMD}=1.14$, $95 \% \mathrm{CI}=0.79-1.50, \quad P<0.001)$. Stratification by assay method showed that serum Cys $C$ levels had significant associations with COPD among the immunonephelometry $(\mathrm{SMD}=0.91,95 \% \mathrm{CI}=0.49-1.33, P<0.001$, Fig. $5 b)$ and ELISA $(\mathrm{SMD}=1.28,95 \% \mathrm{CI}=0.46-2.09$, $P=0.002$ ). In the subgroup analysis of study design, the results showed that COPD in the cohort study had higher serum Cys $\mathrm{C}$ levels $(\mathrm{SMD}=1.90,95 \% \mathrm{CI}=$ $1.55-2.26, P<0.001)$ than that in the case control studies $(\mathrm{SMD}=0.99,95 \% \mathrm{CI}=0.66-1.31, P<0.001)$ and cross sectional study $(\mathrm{SMD}=0.17,95 \% \mathrm{CI}=0.06-0.27$, $P=0.002$, Fig. 5c). Due to the limited number of studies, sensitivity analysis and publication bias analysis were not performed in the subgroup analysis. The impact of heterogeneity slightly decreased for the Asians, immunonephelometry assay, ELISA assay, and case-control, but for the Caucasians, the impact of heterogeneity reduced to $0 \%$. It indicated that ethnicity might be the source of heterogeneity.

\section{Meta-analysis of correlations between serum Cys $\mathrm{C}$ levels and clinical parameters}

We performed the correlation meta-analysis to investigate the relationships of serum Cys $C$ levels with clinical parameters. The results showed that the meta-analysis indicated an inverse correlation between serum Cys $\mathrm{C}$ levels and FEV1\%pre $(\mathrm{Z}=-0.45, \quad 95 \% \mathrm{CI}=-0.58--0.32, \quad P=0.011)$ or FEV1/FVC $(\mathrm{Z}=-0.32,95 \% \mathrm{CI}=-0.50--0.14, P=0.006$; Fig. 6).

\section{Discussion}

This meta-analysis was designed to perform the potential relationship between serum Cys $\mathrm{C}$ levels and patients 


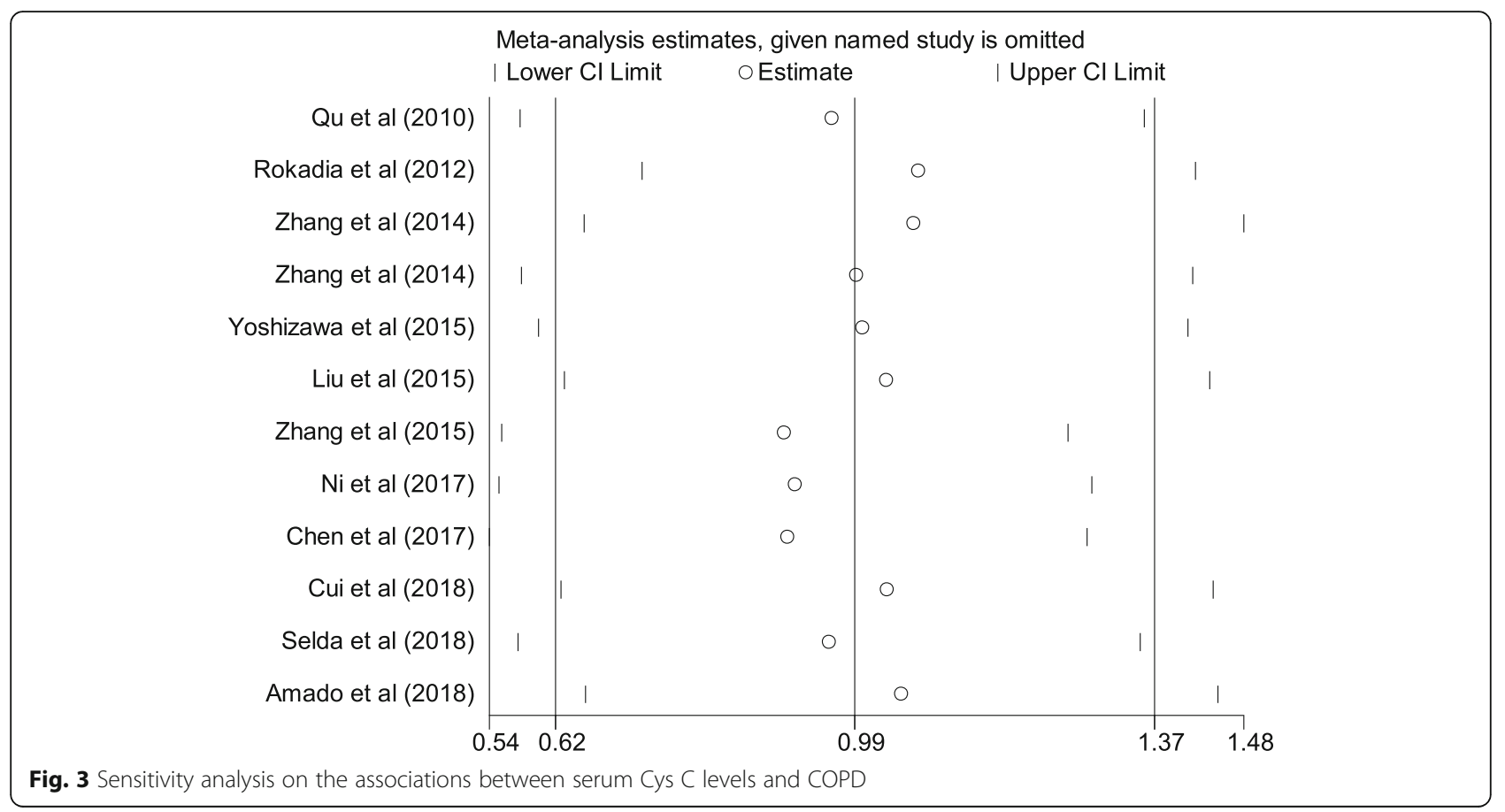

with COPD. The main results of the meta-analysis were as follows:1) the serum levels of Cys $C$ in COPD patients were higher than that in the control; 2) serum Cys $C$ levels in AECOPD patients and SCOPD patients were higher than those in the control group, and the serum Cys $\mathrm{C}$ levels in AECOPD patients were higher than that in stable COPD; 3 ) the serum Cys $\mathrm{C}$ levels were independent of ethnic, assay method and study design; 4) serum Cys $C$ levels had a reverse correlation with FEV1\%pre or FEV1/FVC.
Chronic obstructive pulmonary disease (COPD) is the most common Chronic respiratory disease, and the airflow limitations are associated with long-term chronic inflammatory responses of lung tissues to harmful gases and particles [7]. FEV1\% and the frequency of exacerbations are common indicators for diagnosis and monitoring of treatment response [8]. However, due to the complicated examination of lung function, part of the patients cannot accept lung function as an index to evaluate the therapeutic effect. Therefore, it is necessary

Table 2 Characteristics of studies involving in comparison of the differences of serum Cys C levels

\begin{tabular}{|c|c|c|c|c|c|c|c|c|c|c|}
\hline \multirow[t]{2}{*}{ Author } & \multirow[t]{2}{*}{ Year } & \multicolumn{3}{|c|}{ SCOPD } & \multicolumn{3}{|c|}{ AECOPD } & \multicolumn{3}{|c|}{ Control } \\
\hline & & $\overline{\mathrm{n} 1}$ & Mean1 & SD1 & $\mathrm{n} 2$ & Mean2 & SD2 & $\mathrm{n3}$ & Mean3 & SD3 \\
\hline Qu et al [37] & 2010 & 70 & 1.76 & 0.62 & I & I & I & 30 & 0.98 & 0.51 \\
\hline Sun et al [39] & 2013 & 30 & 0.95 & 0.23 & 54 & 1.12 & 0.26 & / & / & / \\
\hline Zhang et al [27] & 2014 & 129 & 0.115 & 0.05 & / & / & / & 122 & 0.105 & 0.03 \\
\hline Zhang et al [29] & 2014 & 299 & 1.03 & 0.21 & 93 & 1.09 & 0.22 & 151 & 0.84 & 0.18 \\
\hline Yoshizawa et al [40] & 2015 & 108 & 1.2 & 0.4 & / & / & / & 73 & 0.9 & 0.2 \\
\hline Liu et al [41] & 2015 & 60 & 0.1254 & 0.0309 & 72 & 0.1315 & 0.0425 & 36 & 0.1084 & 0.0267 \\
\hline Zhang et al [42] & 2015 & / & / & / & 90 & 1.31 & 0.3 & 90 & 0.87 & 0.13 \\
\hline Zhou et al [43] & 2016 & 60 & 0.94 & 0.22 & 108 & 1.13 & 0.25 & / & / & / \\
\hline $\mathrm{Ni}$ et al [44] & 2017 & 45 & 0.1339 & 0.0154 & 45 & 0.1478 & 0.0212 & 45 & 0.1045 & 0.0209 \\
\hline Selda et al [31] & 2018 & 68 & 1.01 & 0.56 & 58 & 0.97 & 0.29 & 50 & 0.47 & 0.13 \\
\hline Amado et al [28] & 2018 & 65 & 0.97 & 0.32 & / & / & / & 18 & 0.88 & 0.2 \\
\hline Dickson et al [30] & 2019 & 362 & 1.18 & 0.471 & 1637 & 1.21 & 0.47 & / & / & / \\
\hline
\end{tabular}

Abbreviations: COPD Chronic obstructive pulmonary disease, Cys C Cystatin C, AECOPD acute exacerbation COPD, SCOPD stable COPD 


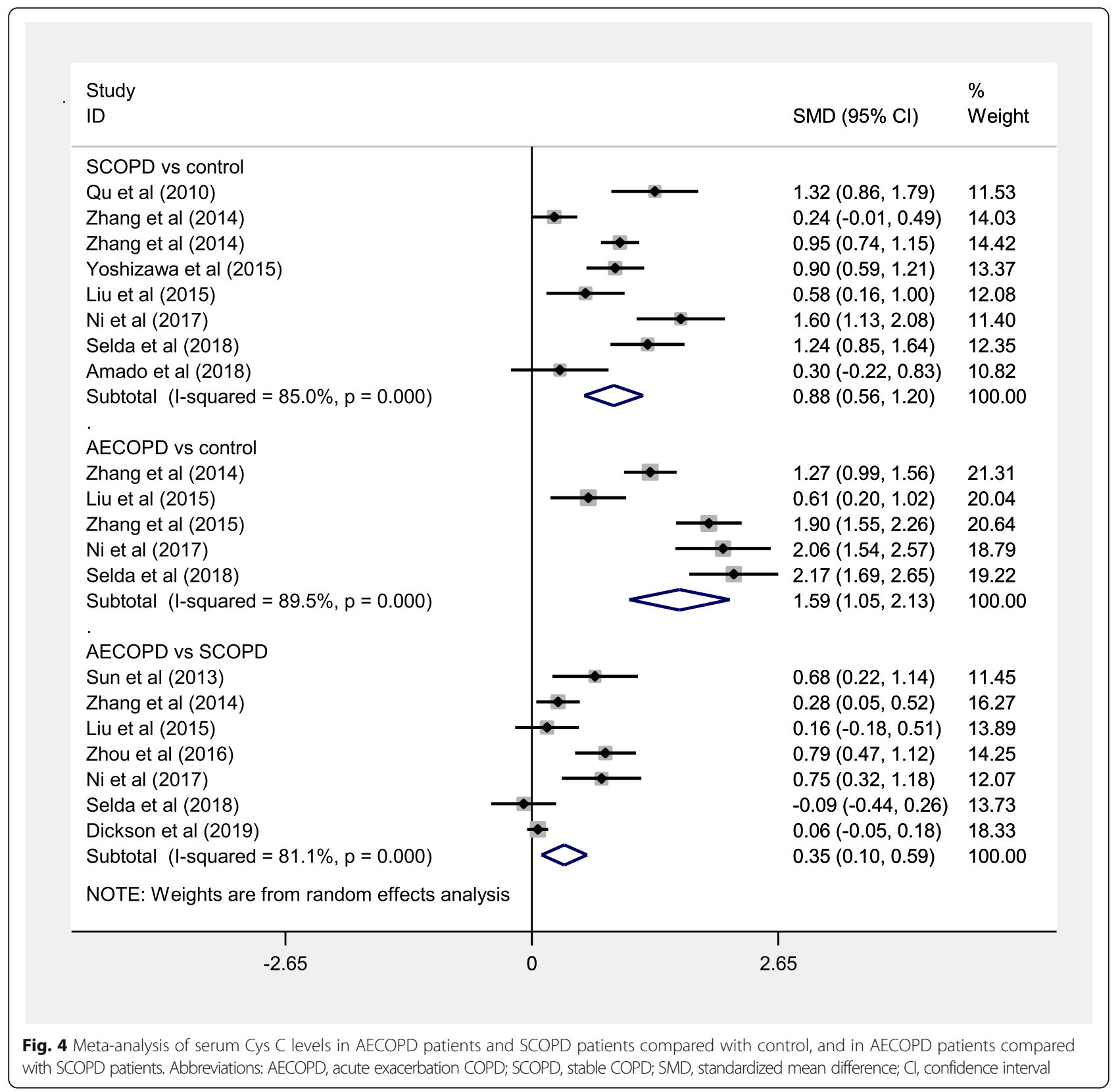

to look for sensitive and reliable markers related to COPD in simple sampling methods, which help clinicians diagnose COPD faster and evaluate its treatment effect more accurately.

The imbalance between protease and antiprotease is one of the important pathogenesis of COPD. Under the action of harmful factors, the protease system is stimulated and activated, which causes the synthesis and secretion of protease to be excessive, and the antiprotease system is destroyed and inactivated, which results that the synthesis or secretion of anti-protease is reduced. These together lead to an increase in the breakdown of matrix proteins in lung tissue, which promotes the development and progression of COPD [2].

Cathepsin is a family of cysteine proteases, and cathep$\sin \mathrm{B}, \mathrm{H}, \mathrm{L}$, and $\mathrm{S}$ are involved in the pathogenesis of COPD [38]. Cys $C$ is a cysteine protease inhibitor, which is the most extensive and effective inhibitor of cathepsin involved in lung tissue destruction [48]. On the one hand, hypoxemia can damage inflammatory cells in the body and releases a large amount of $\mathrm{Cys} C$, which leads to elevated levels of serum Cys $\mathrm{C}$ in patients with COPD [49]. On the other hand, hypoxia causes inflammatory cells such as macrophages to release the cytokines with 
(a)

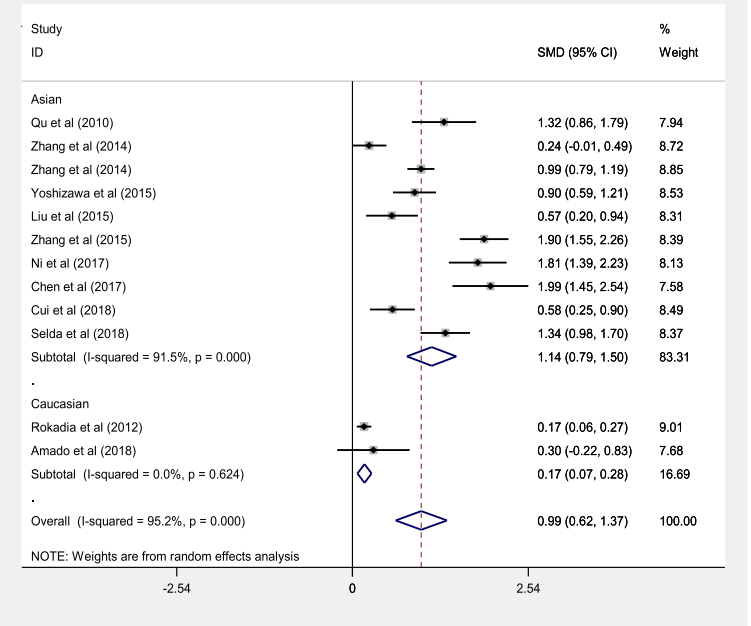

(c)

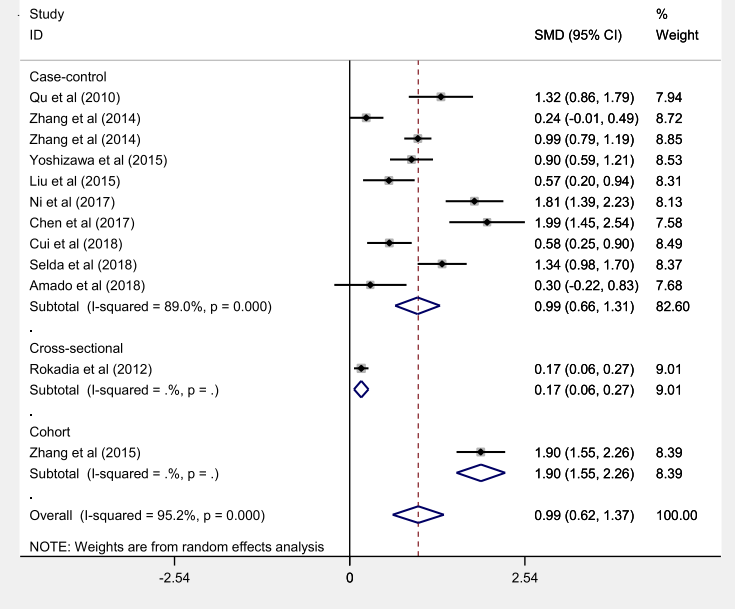

(b)

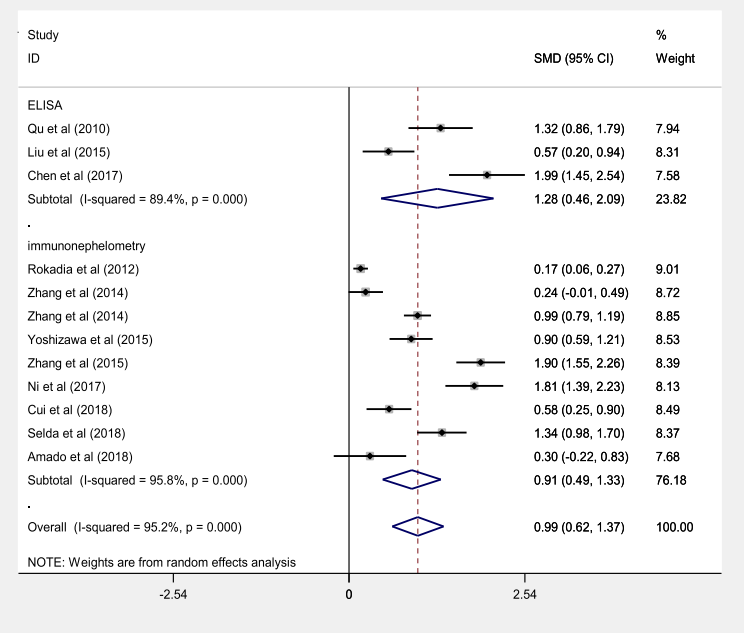

Fig. 5 Meta-analysis of serum Cys C levels in COPD patients compared with controls. Note: Stratified analysis based on ethnicity a, assay methods b, study design c. Abbreviations: SMD, standardized mean difference; Cl, confidence interval; ELISA, enzyme-linked immunosorbent assay

protease activity, which results in the destruction of elastin and leads to emphysema. However, the increasing and activating of protease stimulates the body to synthesize more anti-protease, which will fight against the decomposition and destruction of protease to tissues. Therefore, the levels of Cys $\mathrm{C}$ can predict cathepsin activity indirectly and reliably [26]. Finally, when the body is in a state of hypoxia, the renin-angiotensinaldosterone system (RAAS) and the sympathetic nervous system will be activated, which leads to the contraction of systemic small blood vessels, the decreasing of glomerular blood flow into the aorta, the reducing of effective glomerular filtration rate, and ultimately increasing serum Cys C levels.

This meta-analysis indicated that serum Cys $\mathrm{C}$ levels were higher in COPD. Nakajima et al. [26] reported that serum levels of $\mathrm{Cys} C$ were significantly higher in COPD patients than control. Previous studies showed that inflammatory parameters such as interleukin-6, resistin, tumor necrosis factor, and CRP were significantly correlated with Cys $C$, suggesting that the elevation of Cys $C$ was secondary to the inflammatory processes in the lung $[31,38,50]$. These all above cytokines have been found to be closely related to COPD, which indicated that elevated Cys $C$ levels might be associated with pulmonary inflammation.

Consistent with previous studies, we found that the elevated levels of Cys $\mathrm{C}$ were significantly correlated with disease status and lung function. Hu et al. [24] found that the elevated Cys $\mathrm{C}$ levels was an important and independent risk factor of increased mortality in a hospital setting during COPD exacerbation, Formiga et al. [51] indicated that inspiratory muscle function was reduced with greater degrees of inflammation in COPD as 


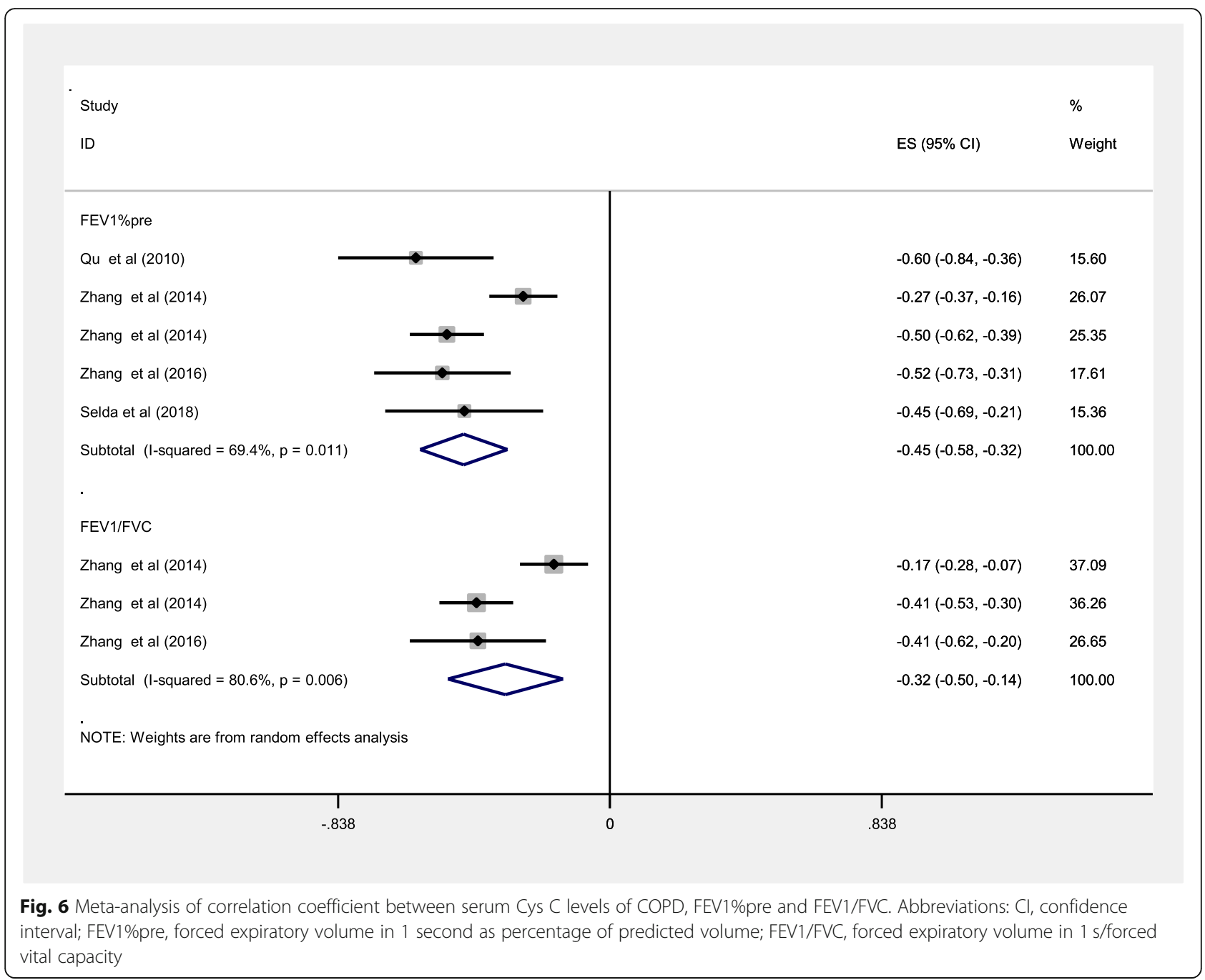

expressed higher levels of Cys C. Combining with the results of previous studies and this meta-analysis, we thought that the elevated levels of serum Cys $C$ might indicate the exacerbation and progression of COPD.

In this meta-analysis, we noted that there was great heterogeneity in the included studies. Moreover, we performed Egger's test, which revealed that there was significant evidence of publication bias in the included studies. Although we used the random-effect model when we found heterogeneity, this increases the probability of type I error. We carried out a sensitivity analysis to assess the stability of this meta-analysis. Sequential removal of each study did not alter the conclusions, suggesting that these results were reliable. We also performed meta-regression analysis and subgroup analysis to perform the possible sources of heterogeneity and reduce the occurrence of type I errors.

Before explaining our results, several limitations of this meta-analysis should be pointed out. First, although some confounding factors might influence the results of the meta-analysis, we did not conduct a subgroup analysis of smoking status due to insufficient original data, the lack of original data also might limit sufficient statistical power to evaluate the potential effects of Cys C levels on the development of COPD. Second, although we tried to gather the original data from the study authors, few authors responded to the data request. Hence, we adopted accepted methods to extract and synthesize data. Third, the concentration of serum Cys $\mathrm{C}$ is affected by a variety of diseases, and a part of these diseases are associated with COPD. Previous studies have indicated that Cys $C$ is strongly associated with renal function [52, 53], chronic hypoxia could also lead to renal dysfunction in patients with COPD [54]. Although patients with kidney disease were excluded when we included the literature, the renal dysfunction caused by COPD might affect the concentration of serum $\mathrm{Cys} C$, which may have some influence on the results. Fourth, the sample sizes in most cited studies were small, and methodologic design may be flawed in smaller studies, the conclusions of smaller 
studies might be impacted by confounding factors, which might have an impact on our results. Fifth, the probability of death due to COPD is much higher in Asian, particularly China, than western countries [55, 56], and Caucasians with COPD have lower serum Cys C level compared with Asians COPD patients [28, 30, $38,42]$. Although we had done a review of the available published studies, most studies included were from Asia, therefore the promotion of conclusions had some limitations. Sixth, many studies have indicated that elevated Cys $\mathrm{C}$ levels may be associated with pulmonary inflammation. However, due to the insufficient data, we did not perform the causal mediation analysis to explore whether serum Cys $C$ was an inflammatory mediator in the development of COPD [57], which made the extension of the results limited. Finally, only published studies were retrieved in this meta-analysis and possible publication bias might exist, and Egger's test found significant evidence of publication bias. Therefore, more keywords should be used to retrieve more studies for further evaluating the relationship between Cys $\mathrm{C}$ levels and COPD.

\section{Conclusion}

Conclusively, the current meta-analysis suggests that, serum Cys $\mathrm{C}$ levels are higher in patients with COPD (both stable COPD and AECOPD) compared to controls. Serum Cys C levels are reversely correlated with FEV1\%pre or FEV1/FVC. The results provided an improved understanding of the roles of Cys $\mathrm{C}$ in COPD development and progression. Further large-scale, unified and well-designed studies are needed to explore the relationship between Cys C and COPD, COPD exacerbation.

\section{Abbreviations}

COPD: Chronic obstructive pulmonary disease; Cys C: Cystatin C; GFR: glomerular filtration rate; SMD: Standardized mean difference; CNKI: China National Knowledge Infrastructure; Cl: Confidence interval; NOS: Newcastle-Ottawa scale; AECOPD: Acute exacerbation COPD; SCOPD: Stable COPD; FEV1\%pre: Forced expiratory volume in 1 second as percentage of predicted volume; FEV1/FVC: Forced expiratory volume in $1 \mathrm{~s} /$ forced vital capacity

\section{Acknowledgements}

None.

\section{Authors' contributions}

LC: study protocol, literature search, data abstraction for meta-analysis, formulation of protocol, writing manuscript. WF, CZ, XY, WS, JW, QW, QZ: literature search, data abstraction for meta-analysis. ML: study protocol, third reviewer of data abstraction, supervision of data abstraction, preparation of manuscript, submission for publication. All authors read and approved the final manuscript.

\section{Funding}

None.

\section{Availability of data and materials}

All data generated or analyzed during this study are included in this published article.
Ethics approval and consent to participate

Not applicable.

\section{Consent for publication}

Not applicable.

\section{Competing interests}

The authors declare that they have no competing interests.

Received: 8 October 2019 Accepted: 3 June 2020

Published online: 26 June 2020

\section{References}

1. Barnes PJ, Burney PG, Silverman EK, Celli BR, Vestbo J, Wedzicha JA, Wouters EF. Chronic obstructive pulmonary disease. Nat Rev Dis Prim. 2015;1:15076.

2. Global Initiative for Chronic Obstructive Lung Disease. Global strategy for the diagnosis, management, and prevention of chronic obstructive pulmonary disease (2018 report). http://www.goldcopd.org/wp-content/ uploads/2017/11/GOLD-2018-v6.0-FINAL-revised-20-Nov_WMS.pdf. Accessed 17 Jan 2018.

3. Vogelmeier CF, Criner GJ, Martinez FJ, Anzueto A, Barnes PJ, Bourbeau J, Celli BR, Chen R, Decramer M, Fabbri LM, et al. Global strategy for the diagnosis, management, and prevention of chronic obstructive lung disease 2017 report: GOLD executive summary. Arch Bronconeumol. 2017;53(3):128-49.

4. Vestbo J, Hurd SS, Agusti AG, Jones PW, Vogelmeier C, Anzueto A, Barnes PJ, Fabbri LM, Martinez FJ, Nishimura M, et al. Global strategy for the diagnosis, management, and prevention of chronic obstructive pulmonary disease: GOLD executive summary. Am J Respir Crit Care Med. 2013;187(4): 347-65.

5. Stolz D, Barandun J, Borer H, Bridevaux PO, Brun P, Brutsche M, Clarenbach C, Eich C, Fiechter R, Frey M, et al. Diagnosis, prevention and treatment of stable COPD and acute exacerbations of COPD: the Swiss recommendations 2018. Resp Int Rev Thor Dis. 2018;96(4):382-98.

6. National Center for Health S: Health, United States. In: Health, United States, 2015: With Special Feature on Racial and Ethnic Health Disparities. edn. Hyattsville (MD): National Center for Health Statistics (US); 2016.

7. Wang C, Xu J, Yang L, Xu Y, Zhang X, Bai C, Kang J, Ran P, Shen H, Wen F, et al. Prevalence and risk factors of chronic obstructive pulmonary disease in China (the China Pulmonary Health [CPH] study): a national crosssectional study. Lancet (London, Engl). 2018;391(10131):1706-17.

8. Global Initiative for Chronic Obstructive Lung Disease. Global strategy for the diagnosis, management, and prevention of chronic obstructive pulmonary disease (2017 report). https://goldcopd.org/wp-content/ uploads/2017/02/wms-GOLD-2017-FINAL.pdf. Accessed 17 Jan 2018.

9. Han MK, Kim MG, Mardon R, Renner P, Sullivan S, Diette GB, Martinez FJ. Spirometry utilization for COPD: how do we measure up? Chest. 2007; 132(2):403-9.

10. Ding B, Judge D, Small M, Bent-Ennakhil N, Siddiqui S. Functional performance in patients with COPD: association with treatment regimen, GOLD group, lung function, and symptom burden in a cross-sectional study. Int J Chronic Obstruct Pulmon Dis. 2018;13:2785-96.

11. Zi M, Xu Y. Involvement of cystatin C in immunity and apoptosis. Immunol Lett. 2018;196:80-90.

12. Georges S, Ruiz Velasco C, Trichet V, Fortun Y, Heymann D, Padrines M. Proteases and bone remodelling. Cytokine Growth Factor Rev. 2009;20(1): 29-41.

13. Tavera C, Prevot D, Girolami JP, Leung-Tack J, Colle A. Tissue and biological fluid distribution of cysteine proteinases inhibitor: rat cystatin C. Biol Chem Hoppe Seyler. 1990;371(Suppl):187-92.

14. Dharnidharka VR, Kwon C, Stevens G. Serum cystatin C is superior to serum creatinine as a marker of kidney function: a meta-analysis. Am J Kidney Dis. 2002;40(2):221-6.

15. Laterza OF, Price CP, Scott MG. Cystatin C: an improved estimator of glomerular filtration rate? Clin Chem. 2002;48(5):699-707.

16. Elsayed MS, El Badawy A, Ahmed A, Omar R, Mohamed A. Serum cystatin C as an indicator for early detection of diabetic nephropathy in type 2 diabetes mellitus. Diab Metab Syndrome. 2019;13(1):374-81.

17. Meng L, Yang Y, Qi LT, Wang XJ, Xu GB, Zhang BW. Elevated serum cystatin $\mathrm{C}$ is an independent predictor of cardiovascular events in people with relatively normal renal function. J Nephrol. 2012;25(3):426-30. 
18. Tantawy AAG, Adly AAM, Ismail EAR, Abdelazeem M. Clinical predictive value of Cystatin C in pediatric sickle cell disease: a marker of disease severity and subclinical cardiovascular dysfunction. Cl Appl Thrombosis Hemostasis. 2017:23(8):1010-7.

19. Kato K, Takata Y, Usui Y, Shiina K, Asano K, Hashimura Y, Saruhara H, Nishihata Y, Tomiyama H, Yamashina A. Severe obstructive sleep apnea increases cystatin C in clinically latent renal dysfunction. Respir Med. 2011; 105(4):643-9.

20. Archontogeorgis K, Nena E, Tsigalou C, Voulgaris A, Xanthoudaki M, Froudarakis M, Steiropoulos P: Cystatin C levels in middle-aged patients with obstructive sleep apnea syndrome. Pulm Med 2016, 2016:8081723.

21. Suzuki K, Furuse H, Tsuda T, Masaki Y, Okazawa S, Kambara K, Inomata M, Miwa T, Matsui S, Kashii T, et al. Utility of creatinine/cystatin C ratio as a predictive marker for adverse effects of chemotherapy in lung cancer: a retrospective study. J Int Med Res. 2015:43(4):573-82

22. Werle B, Sauckel K, Nathanson CM, Bjarnadottir M, Spiess E, Ebert W, Abrahamson M. Cystatins C, E/M and F in human pleural fluids of patients with neoplastic and inflammatory lung disorders. Biol Chem. 2003;384(2): 281-7.

23. Takeyabu K, Betsuyaku T, Nishimura M, Yoshioka A, Tanino M, Miyamoto K, Kawakami Y. Cysteine proteinases and cystatin C in bronchoalveolar lavage fluid from subjects with subclinical emphysema. Eur Respir J. 1998;12(5): 1033-9.

24. Hu G, Wu Y, Zhou Y, Yu Y, Liang W, Ran P. Cystatin C as a predictor of inhospital mortality after exacerbation of COPD. Respir Care. 2016;61(7):950-7.

25. Perez-Calvo JI, Sanchez-Marteles M, Ruiz-Ruiz FJ, Morales-Rull JL, NietoRodriguez JA. Differential prognostic utility of NTproBNP and Cystatin C in patients with acute exacerbation of chronic pulmonary disease. JRSM Short Rep. 2010;1(5):44.

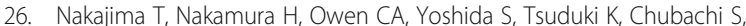
Shirahata T, Mashimo S, Nakamura M, Takahashi S, et al. Plasma Cathepsin S and Cathepsin S/Cystatin C ratios are potential biomarkers for COPD. Dis Markers. 2016;2016:4093870.

27. Zhang M, Fu SH, Cui H, Zhu BP, Liu L, Wang DL. Serum cystatin C and indices of lung function in elderly Chinese men with chronic obstructive pulmonary disease. Aging Clin Exp Res. 2014;26(2):193-9.

28. Amado CA, Garcia-Unzueta MT, Lavin BA, Guerra AR, Aguero J, Ramos L, Munoz P. The ratio serum Creatinine/serum Cystatin C (a surrogate marker of muscle mass) as a predictor of hospitalization in chronic obstructive pulmonary disease outpatients. Respir Int Rev Thor Dis. 2018:1-8.

29. Zhang Y, Zhu Y, Wu Y, Wang G, Xie X, Ke R, Li S, Liu L, Fen W, Li F, et al. Serum cystatin $\mathrm{C}$ as a potential biomarker for the evaluation COPD. Int J Clin Exp Med. 2014;7(12):5484-90.

30. MINJA DA: Role of cystatin C and N-Terminal pro-BNP in hospitalized COPD patient with preserved LEFT ventrucular ejection fraction. master. Shandong University; 2019.

31. Telo S, Kuluozturk M, Deveci F, Kirkil G, Oner O, Kaman D. Serum CYSTATIN c levels in COPD: potential diagnostic value and relation between respiratory functions. J Med Biochem. 2018;37(4):434-40.

32. Chen B, Benedetti A. Quantifying heterogeneity in individual participant data meta analysis with binary outcomes. Syst Rev. 2017;6(1):243.

33. Wan X, Wang W, Liu J, Tong T. Estimating the sample mean and standard deviation from the sample size, median, range and/or interquartile range. BMC Med Res Methodol. 2014;14:135.

34. Wikipedia. Fisher transformation. http://www.en.wikipedia.org/wiki/Fisher transformation. Accessed 17 July 2019.

35. Egger M, Davey Smith G, Schneider M, Minder C. Bias in meta-analysis detected by a simple, graphical test. BMJ (Clinical research ed). 1997; 315(7109):629-34.

36. Begg CB, Mazumdar M. Operating characteristics of a rank correlation test for publication bias. Biometrics. 1994;50(4):1088-101.

37. Qu S, Liu J, Zeng W, Fu X, Yang S. Diagnostic values of serum Cystatin C in early renal damage of chronic obstructive pulmonary diseases. J Clin Internal Med. 2010;27(10):677-9.

38. Rokadia HK, Agarwal S. Serum Cystatin C and emphysema: results from the National Health and nutrition examination survey (NHANES). Lung. 2012; 190(3):283-90

39. Sun Z, Cheng Y, Lu Z. Relationship between Cystatin C and acute exacerbation of chronic obstructive pulmonary disease. Chin Foreign Med Res. 2013;27:1-2,3.
40. Yoshizawa T, Okada K, Furuichi S, Ishiguro T, Yoshizawa A, Akahoshi T, Gon Y, Akashiba T, Hosokawa Y, Hashimoto S. Prevalence of chronic kidney diseases in patients with chronic obstructive pulmonary disease: assessment based on glomerular filtration rate estimated from creatinine and cystatin C levels. Int J COPD. 2015;10:1283-9.

41. Liu J, Yu A. Detection of indicators of early renal injury in patients with COPD and its clinical significance. Modern Pract Med. 2015;27(11):14541455,1473 .

42. Zhang M, Li Y, Yang X, Shan H, Zhang Q, Ming Z, Xie Y, Chen H, Liu Y, Zhang J. Serum Cystatin $C$ as an inflammatory marker in exacerbated and convalescent COPD patients. Inflammation. 2016;39(2):625-31.

43. Zhou $\mathrm{Q}$, Wang $\mathrm{S}$. The role of Cys-C in assessing the recovery of AECOPD patients. Pract Prevent Med. 2016;23(5):598-600.

44. Ni H, Gu H, Wang $H$, Yang W. Detection of indicators of early renal injury in patients with COPD and its clinical significance. Clin Med China. 2017;33(7): 614-7.

45. Chen $Y$, Jia Q. Correlation analysis of early renal injury with HIF-1a, Cys C, and B2-MG levels in COPD patients. J Clin Res. 2017;34(7):1294-1296,1300.

46. Cui $S$, Yang R. Changes and significance of serum Cys-C level in patients with COPD complicated with pulmonary hypertension. J Clin Pulmon Med. 2018;23(1):32-34,37.

47. Duval S, Tweedie R. Trim and fill: a simple funnel-plot-based method of testing and adjusting for publication Bias in Meta-analysis. Biometrics. 2000; 56(2):455-63.

48. Abboud RT, Vimalanathan S. Pathogenesis of COPD. Part I. the role of protease-antiprotease imbalance in emphysema. Int J Tuberc Lung Dis. 2008;12(4):361-7.

49. van Deursen VM, Damman K, Voors AA, van der Wal MH, Jaarsma T, van Veldhuisen DJ, Hillege HL. Prognostic value of plasma neutrophil gelatinaseassociated lipocalin for mortality in patients with heart failure. Circ Heart Failure. 2014;7(1):35-42.

50. Balta S, Demirkol S, Ay SA, Cakar M, Sarlak H, Celik T. Serum cystatin-C levels correlate with endothelial dysfunction in patients with the metabolic syndrome. J Internal Med. 274(2):200-1.

51. Formiga MF, Vital I, Urdaneta G, Masters B, Herrera J, Campos MA, Cahalin LP. Higher serum levels of systemic inflammatory markers are linked to greater inspiratory muscle dysfunction in COPD. Clin Respir J. 2019;13(4): 247-55.

52. Zhang Z, Lu B, Sheng $X$, Jin N. Cystatin C in prediction of acute kidney injury: a systemic review and meta-analysis. Am J Kidney Dis. 2011;58(3): 356-65.

53. Björk J, Nyman U, Berg U, Delanaye P, Dubourg L, Goffin K, Grubb A, Hansson M, Littmann K, Åsling-Monemi K, Bökenkamp A, Pottel H. Validation of standardized creatinine and cystatin C GFR estimating equations in a large multicentre European cohort of children. Pediatr Nephrol. 2019;34(6):1087-98.

54. Yoshizawa T, Okada K, Furuichi S, Ishiguro T, Yoshizawa A, Akahoshi T, Gon Y, Akashiba T, Hosokawa Y, Hashimoto S. Prevalence of chronic kidney diseases in patients with chronic obstructive pulmonary disease: assessment based on glomerular filtration rate estimated from creatinine and cystatin C levels. Int J Chron Obstruct Pulmon Dis. 2015;10:1283-9.

55. López-Campos JL, Tan W, Soriano JB. Global burden of COPD. Respirology. 2016;21(1):14-23.

56. Yin P, Wang H, Vos T, Li Y, Liu S, Liu Y, Liu J, Wang L, Naghavi M, Murray CJ, et al. A subnational analysis of mortality and prevalence of COPD in China from 1990 to 2013: findings from the global burden of disease study 2013. Chest. 2016;150(6):1269-80.

57. Zhang Z, Zheng C, Kim C, Van Poucke S, Lin S, Lan P. Causal mediation analysis in the context of clinical research. Ann Transl Med. 2016;4(21):425

\section{Publisher's Note}

Springer Nature remains neutral with regard to jurisdictional claims in published maps and institutional affiliations. 